\title{
Association of CYP1A1, GSTM1 and GSTT1 gene polymorphisms with risk of prostate cancer in Algerian population
}

\author{
Somia Medjani ${ }^{1,2,3^{*}}$, Djalila Chellat-Rezgoune ${ }^{1,2}$, Taher Kezai ${ }^{3}$, Mohammed Chidekh ${ }^{3}$, Noureddine Abadi ${ }^{2}$
} and Dalila Satta ${ }^{1,2}$

\begin{abstract}
Background: Prostate cancer is the most common cancer in the world, and its etiology involves the interaction of genetic and environmental factors. Interindividual differences observed in the metabolism of xenobiotics may be due to polymorphisms of genes encoding the detoxification enzymes. This genetic variability seems to be associated with differences in susceptibility to certain types of cancers, including prostate cancer. Our study has been made in order to investigate a possible genetic predisposition to prostate cancer in an Algerian population, through the analysis of genetic polymorphisms of three enzymes metabolizing xenobiotics namely cytochrome P450 (CYP) 1A1, glutathione S-transferase mu 1 (GSTM1) and GST theta 1 (GSTT1).
\end{abstract}

Methods: The current case-control study included 101 prostate cancer patients and 101 healthy controls. Genotyping of CYP1A1 T3801C polymorphisms and GSTM1/GSTT-null was made, respectively, by PCR-RFLP and multiplex PCR.

Results: No significantly positive associations were found for the CYP1A1 T3801C $[p=0.71, \mathrm{OR}=1.23(0.56-2.72)]$ and GSTM1-null $[p=0.26, \mathrm{OR}=1.37(0.76-2.4)]$ polymorphisms and prostate cancer susceptibility. However, we detect a highly significant association between GSTT1-null genotype $[p=0.03, \mathrm{OR}=2.03(1.06-3.99)]$, GSTM1/GSTT1-double null genotype $[p=0.027, \mathrm{OR}=2.6 ; \mathrm{Cl}(1.07-6.5)]$ and prostate cancer risk. Furthermore, no statistically significant differences between the studied polymorphisms and tumor parameters (the Gleason score and clinical stages of aggressiveness) at diagnosis of PCa.

Conclusions: The risk of developing prostate cancer in Algeria does not appear to be associated with CYP1A1 T3801C genotypes and GSTM1-null, but GSTT1-null and GSTM1/GSTT1-double null genotypes increased the risk of prostate cancer.

Keywords: Prostate cancer, Genetic polymorphism, Cytochrome P4501A1 T3801C, Glutathione S-transferase (GST), GSTM1, GSTT, PCR-RFLP, multiplex PCR

\section{Introduction}

Prostate cancer ( $\mathrm{PCa}$ ) is considered as a major public health problem in the world because of its increased mortality and morbidity rates. It is evaluated as a most

\footnotetext{
*Correspondence: soumia.medjani@umc.edu.dz

1 Department of Animal Biology, Laboratory of Molecular and Cellular Biology, Faculty of Natural and Life Sciences, University of Frères Mentouri Constantine 1, 25000 Constantine, Algeria

Full list of author information is available at the end of the article
}

frequently diagnosed solid neoplasm. Overall, $\mathrm{PCa}$ is the second major cause of cancer-related death among men (Europeans, Americans and to some extent in Africans as well) $[1,2]$. In Algeria, it is the most common male cancer (10\% of cancers in men) with 1645 new cases with an average incidence of 10.8/100,000 [3]. According to Globocan 2018, PCa is the third most common cancer in men with 2578 new cases.

In order to understand the biology, etiology and to develop new interventions of PCa, complete analysis of 
different risk factors along with their comparison with genetics is essential [4].

Clinical and epidemiological data suggest that the development of $\mathrm{PCa}$ is a multiphase process. [5]. Smoking, dietary habits, lifestyle factors, environmental factors, as well as geographical/racial factors and genetics factors may be involved in PCa development. Also, modification in carcinogen metabolism genes may play a critical role in PCa development due to their activation or detoxification functions [6].

Xenobiotic-metabolizing enzymes $(X M E)$, coded by a family of xenobiotic-metabolizing genes $(X M G)$, have a major role in elimination of many xenobiotics or environmental chemical compounds from the body. This mechanism of detoxification occurs in two phases [7]. In phase I, cytochrome P450 enzymes play an important role in the metabolism of estrogen and polycyclic aromatic hydrocarbons (PAHs). They catalyze the activation of procarcinogenic PAHs and their dysfunctions can cause damage to DNA, leading to carcinogenesis.

The CYP1A1 gene is located on 15q22-q24 [8], contains 7 exons and 6 introns and spans 5810 base pairs. Eleven polymorphisms of CYP1A1 have been described, four of which have been the most studied for their involvement in modifying the risk of carcinogenesis: $m 1$ (T3801C), $m 2$ (A2455G), $m 3$ (T3205C) and $m 4$ (C2453A) [9]. The CYP1A1 3801T/C (also named MspI polymorphism, "2A or $\mathrm{m} 1$ ), results from a replacement of thymine by a cytosine at the 3801st base pair in the 3' flanking region of the gene $[9,10]$.

Glutathione S-transferases (GSTS) involve a superfamily of multifunctional and ubiquitous phase II metabolic enzymes. It catalyzes the conjugation of electrophilic substrates to soluble glutathione to facilitate their cellular excretion. Additionally, GSTs are able to detoxify noxious products of the cellular metabolism, such as reactive oxygen and nitrogen species through their glutathione peroxidase activity $[11,12]$. GSTs constitute the major antioxidant defensive system against oxidative stress by reducing reactive oxygen species, which are generated by many toxic xenobiotics [13].

In human, these enzymes are found in cytosol, microsome and mitochondria. The superfamily of genes encoding cytosolic glutathione S-transferases consists of eight classes: $\alpha$ (GSTA), $\kappa$ (GSTK), $\mu$ (GSTM), $\omega$ (GSTO), $\pi$ (GSTP), $\varsigma$ (GSTS), $\theta$ (GSTT) and $\zeta$ (GSTZ) [14]. GSTM1 and GSTT1 genes are situated on chromosome 1p13.3 and 22q11.23, respectively [15]. GSTM1 preferentially detoxifies carcinogens which are found in tobacco (epoxides and hydroxylated derivatives), whereas GSTT1 is specific for the biotransformation of many smaller toxins derived from tobacco smoke such as butadiene and ethylene oxides [16].
Homozygous deletions of the GSTM1 and GSTT1 genes are common and result in a complete loss of enzymatic activity. Hence, individuals are at greater risk toward the development of malignancies $[17,18]$.

Previous studies have been reported regarding the genetic status of GSTM1 and GSTT1 polymorphism and prostate cancer development. But the effect of polymorphisms of these two genes on PCa is still unclear because of inconsistent results among different populations [19].

The aim of the present study was to assess the influence of CYP1A1 and glutathione S-transferases (GSTM1, GSTT1) on the risk of PCa.

\section{Methods}

\subsection{Subjects}

The study population consisted of 101 patients with prostate cancer and 101 healthy controls.

All patients were histologically diagnosed with prostate cancer; thus, all stages of this tumor development have been included. Cases were recruited from the Uro-Nephrology Hospital "the Department of Urology and Renal Transplantation," Constantine, Algeria. The criteria for patient inclusion was a suspicious finding upon digital rectal examination (DRE) and/or elevated serum levels of PSA ( $>4 \mathrm{ng} / \mathrm{ml}$ ) followed by a histopathologic confirmation of prostate cancer.

Control subjects were volunteers, healthy men, without a family history of malignant disease. Their PSA levels were within the normal limit $(<4 \mathrm{ng} / \mathrm{mL})$ and showed no signs of prostate hyperplasia or prostate carcinoma by DRE.

A detailed questionnaire was elaborated and used to obtain study information's.

\subsection{Ethics statement}

Our research has been approved by the local Ethics Committee. The use of human blood sample and the protocol in this study strictly conformed to the principles expressed in the Declaration of Helsinki, and informed (written) consent was obtained from all participants.

\section{Molecular analysis of CYP1A1, GSTM1 and GSTT1 gene polymorphisms}

\subsection{Blood collection and DNA isolation}

Blood samples $(10 \mathrm{ml})$ were obtained from peripheral veins of patients and controls in vacutainer K3EDTA tubes. Genomic DNA isolation followed a standard procedure for $\mathrm{NaCl}$ extraction and ethanol precipitation. Genomic DNA samples were stored at $-20^{\circ} \mathrm{C}$ until use. 


\subsection{CYP1A1 T3801C genotyping}

Detection of CYP1A1 T3801C polymorphism was performed by polymerase chain reaction restriction fragment length polymorphism (PCR-RFLP).

One set of forward 5'-GGCTGAGCAATCTGACCC TA-3 and reverse: 5'-TAGGAGTCTTGTCTCATGCCT$3^{\prime}$ primers were used for the amplification of a fragment of $340 \mathrm{pb}$.

DNA was amplified in a total volume of $20 \mu \mathrm{l}$, containing $\mathrm{H}_{2} \mathrm{O}, \mathrm{MgCl}_{2}(50 \mathrm{mM})$, primers (100 $\left.\mathrm{ng} / \mu \mathrm{l}\right)$, dNTP $(0,2 \mathrm{mM})$, PCR buffer $10 \mathrm{X}$ and Taq polymerase (Taq DNA $5 \mathrm{U} / \mu \mathrm{l})$. For each individual, $2 \mu \mathrm{l}$ of DNA (20 à $50 \mathrm{ng} / \mu \mathrm{l}$ ) is mixed with $18 \mu \mathrm{l}$ of the mixture in a PCR tube.

Following an initial denaturation step at $94{ }^{\circ} \mathrm{C}$ for $4 \mathrm{~min}$. Thirty-seven cycles of amplification were carried out, denaturation $\left(94{ }^{\circ} \mathrm{C}\right.$ for $\left.30 \mathrm{~s}\right)$, annealing $\left(60{ }^{\circ} \mathrm{C}\right.$ for $30 \mathrm{~s})$ and extension $\left(72{ }^{\circ} \mathrm{C}\right.$ for $\left.30 \mathrm{~s}\right)$, with a final extension step at $72{ }^{\circ} \mathrm{C}$ for $10 \mathrm{~min}$. The amplified products were electrophoresed on a $2 \%$ agarose gel to check PCR product size. The PCR amplicons generated for $\mathrm{m} 1$ (340 bp) were subjected to restriction digestion. Msp1 restriction enzyme was used to detect polymorphisms in the $C Y P 1 A 1 \mathrm{~m} 1$. The reaction mixtures were incubated at $37{ }^{\circ} \mathrm{C}$ for $12 \mathrm{~h}$, electrophoresed on $3 \%$ agarose gel and stained with ethidium bromide for visualization.

\subsection{GSTM1 and GSTT1 genotyping}

The GSTM1 and GSTT1 gene deletions were analyzed simultaneously by multiplex PCR. $\beta$-globulin was used as an internal control, confirming successful PCR amplification to ensure that the GSTM1-null and GSTT1-null were due to deletion of GST alleles and not due to failure of the PCR.

To detect the GSTM1 deletion, the primers used were GSTM1 F (5'-GAACTCCCTGAAAAGCTAAAGC-3') and GSTM1 R (5'-GTTGGGCTCAAATATACGGTGG$\left.3^{\prime}\right)$. For GSTT1, the primers used were GSTT1 F (5'-TTC CTTACTGGTCCTCACATCTC-3') and GSTT1R (5'TCACCGGATCATGGCCACCA- $\left.3^{\prime}\right)$; For $\beta$-globulin $\mathrm{F}$ (5'-ACA CAA CTG TGT TCA CTA GC-3') and R (5'CAA CTT CAT CCA CGT TCA CC-3').

The PCR conditions were: 4 min of initial denaturation at $95{ }^{\circ} \mathrm{C}$, followed by of 37 cycles of denaturation at $94{ }^{\circ} \mathrm{C}$ for $1 \mathrm{~min}$, annealing at $62{ }^{\circ} \mathrm{C}$ for $1 \mathrm{~min}$ and extension at $72{ }^{\circ} \mathrm{C}$ for $1 \mathrm{~min}$ with a final extension at $72{ }^{\circ} \mathrm{C}$ for $4 \mathrm{~min}$.

The GSTM1 fragment was $230 \mathrm{bp}$, the GSTT1 fragment was $480 \mathrm{bp}$, and the $\beta$-globulin fragment was $110 \mathrm{bp}$ in size.

\subsection{Statistical analysis}

Statistical analysis was carried out using $\mathrm{R}$ software version 3.2.3. Statistical significance of differences in genotype frequencies between patients and controls was estimated by the Chi-square test. Logistic regression was used to evaluate the effect of genotypes, after adjusting age and tobacco. We also performed a logistic regression analysis to determine whether the clinical stage and the pathological grade were associated with PCa risk. The odds ratio (OR) and its $95 \%$ confidence interval (CI) were used to illustrate the association, with $p<0.05$ considered statistically significant in all tests.

\section{Results}

Relevant characteristics of cases and controls are given in Table 1. In our study, patients and controls were aged between 50 and 89 years, and the average age of prostate cancer patients and controls was $70.66 \pm 8.32$ and $68 \pm 9.19$, respectively. No statistically significant difference was observed between cases $(63.37 \%)$ and controls $(55.37 \%)$ regarding the smoking status $(p=0.25)$. Also, the majority of patients population have higher total PSA rate and diagnosed at advanced stage. There was no significant interaction between CYP1A1, GSTM1, GSTT1genotypes and smoking (data not shown).

Representative CYP1A1 T3801C genotyping results are summarized in Fig. 1. Table 2 shows the distribution of allelic and genotypic frequencies for the polymorphism CYP1A1 T3801C between individuals with and without prostate cancer and their relation with risk of prostatic carcinogenesis.

A total of $101 \mathrm{PCa}$ patients and 101 control subjects were included in this study. Frequencies of $C Y P 1 A 1$ T3801C polymorphism genotypes show that the wild genotype $(T T)$ is the most common in both populations. $82(81.18 \%)$ cases and $85(84.15 \%)$ of controls were $(T T)$. Heterozygote genotype (TC) was found in 19 (18.81\%) patients and $16(15.84 \%)$ normal controls. Thus, no mutated genotype (CC) was identified in all genotyped individuals. These results suggest that no statistically significant association of CYP1A1 T3801C gene polymorphism with $\mathrm{PCa}$ in the tested population $[p=0.71$, $\mathrm{OR}=1.23(0.56-2.72)$ ].

Representative GSTM1 and GSTT1 genotyping results are illustrated in Fig. 2. Table 3 presents genotype frequencies for the GSTM1 and GSTT1.

GSTM1 and GSTT1 genotypes distribution among all population (cases and controls), as well as the estimates of PCa risk, are summarized in Table 3.

GSTM1 was deleted in $50.49 \%$ of controls and $42.57 \%$ of PCa patients. No significant association was found when comparing GSTM1 gene deletions $(p=0.26$, $\mathrm{OR}=1.37$, CI [0.76-2.4]) with risk of PCa.

GSTT1-null genotype was observed in $37.62 \%$ of patients with PCa and $22.77 \%$ of controls subjects, which 
Table 1 Characteristics of the study population

\begin{tabular}{llll}
\hline Characteristics & Cases $\boldsymbol{N}(\%)$ & Controls $\boldsymbol{N}(\%)$ & OR [IC] \\
\hline Sample size & 101 & 101 & \\
Age (mean years \pm SD) & $70.66 \pm 8.32$ & $68.96 \pm 9.19$ & \\
Smoking status & & $45(44.45)$ & $1.39[0.8,2.4]$ \\
Non-smoker & $37(36.63)$ & $56(55.44)$ & \\
Smoker & $64(63.37)$ & & \\
Clinical criteria & & \\
Total PSA Rate $(n g / m l)$ & & \\
PSA $\leq 10$ & $8(08.00)$ & \\
$10<$ PSA $<50$ & $29(38.67)$ & \\
PSA $\geq 50$ & $64(53.33)$ & \\
mean ( \pm SD) & $74.49 \pm 33.77$ & \\
The Gleason score & & \\
GS $\leq 7$ & $37(36.63)$ & \\
GS $>7$ & $64(63.36)$ & \\
Clinical stage & & \\
Localized & $42(41.58)$ & \\
Advanced & $59(58.41)$ & \\
\hline
\end{tabular}

SD Standard deviation, PSA Prostate-specific antigen, GS Gleason score

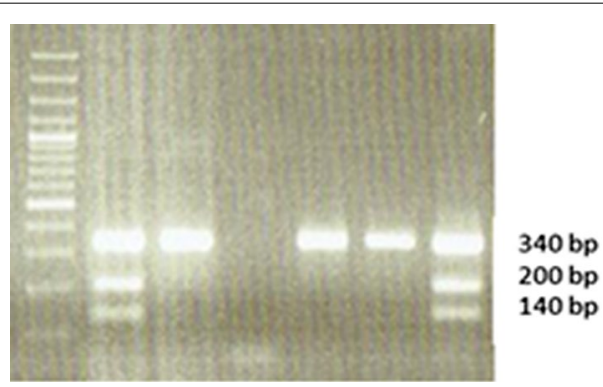

Fig. $13 \%$ agarose gel illustrating the amplifications of CYP1A1 polymorphism. DNA fragments digested with Msp 1 and electrophoresis were TT homozygous major type which reveals the uncut amplicon of 340 pb; CC homozygous minor type (mutant) should show two fragments (200 and 140 bp), while the heterozygous genotype $T / C$ presents three fragments: the uncut fragment $340 \mathrm{bp}$ and two restriction fragments of 200 and $140 \mathrm{bp}$
Table 2 Statistical analysis and distribution of CYP1A1 T3801C genotypes and allelic frequencies for PCa patients and controls

\begin{tabular}{lllll}
\hline & Patients (\%) & Controls (\%) & OR/Cl & P value \\
\hline TT & $82(81.18)$ & $85(84.15)$ & & \\
TC & $19(18.81)$ & $16(15.84)$ & $1.23[0.56-2.72]$ & 0.71 \\
CC & 0 & 0 & $/$ & $/$ \\
TC + CC vs TT & $1(18.81)$ & $16(15.84)$ & $1.18[1.72-2.42]$ & 0.71 \\
TT+TC vs CC & $101(100)$ & $101(100)$ & $/$ & $/$ \\
Allele T & $183(90.59)$ & $186(92.07)$ & & $/$ \\
Allele C & $38(9.41)$ & $32(7.92)$ & $1.20[0.57-2.55]$ & 0.72 \\
\hline
\end{tabular}

$O R$ odds ratios, $\mathrm{Cl}$ confidence intervals

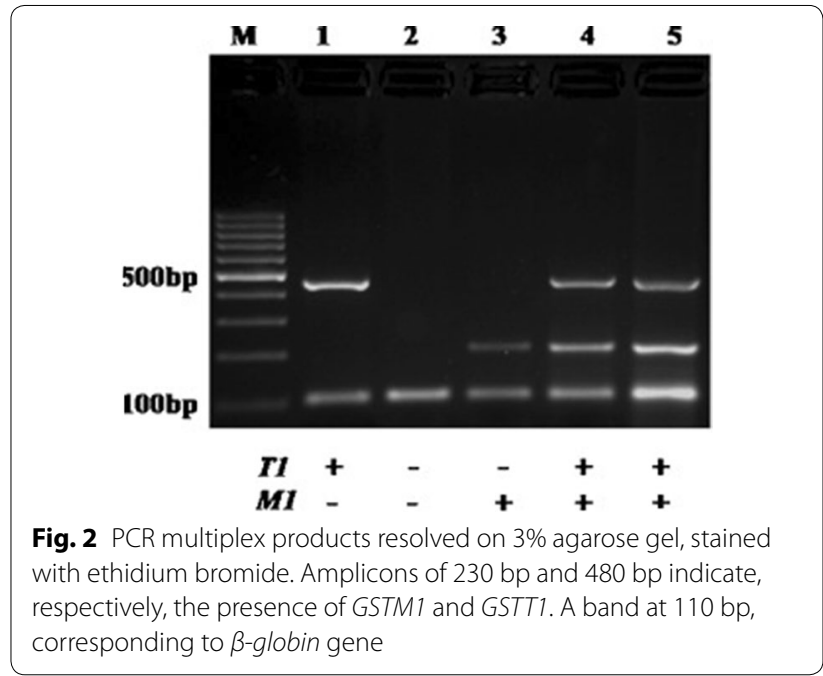

was statistically significant $[p=0.03, \quad \mathrm{OR}=2.03, \quad \mathrm{CI}$ (1.06-3.99)].

Combined frequencies of GSTM1 and GSTT1 polymorphisms Wild/Wild $(+/+)$, Wild/Null $( \pm)$, Null/ Wild (干) and Null/Null (-/-) in healthy control were $40.59,9.90,36.63$ and $12.87 \%$, respectively, whereas in PCa patients, the frequencies were 29.70, 12.87, 32.67 and $24.75 \%$, respectively. The significant association was found between GSTM1-null/GSTT1-null (double null) $(p=0.027)[\mathrm{OR}=2.6$; $\mathrm{CI}(1.07-6.5)]$. 
Table 3 GSTM1, GSTT1 genotypes distribution among tested patients and healthy controls and susceptibility of PCa

\begin{tabular}{lllll}
\hline & Patients \% & Controls \% & OR/Cl & P value \\
\hline$M+$ & $43(42.57)$ & $51(50.49)$ & $/$ & $/$ \\
$M-$ & $58(57.42)$ & $50(49.50)$ & $1.37[0.76-2.4]$ & 0.26 \\
$T+$ & $63(62.37)$ & $78(77.22)$ & $/$ & $/$ \\
$T-$ & $38(37.62)$ & $23(22.77)$ & $2.03[1.06-3.99]$ & 0.03 \\
$M+/ T+$ & $30(29.70)$ & $41(40.59)$ & $/$ & $/$ \\
$M+/ T-$ & $13(12.87)$ & $10(9.90)$ & $1.76[0.62-5.2]$ & 0.33 \\
$M-/ T+$ & $33(32.67)$ & $37(36.63)$ & $1.21[0.60-2.5]$ & 0.61 \\
$M-/ T-$ & $25(24.75)$ & $13(12.87)$ & $2.6[1.07-6.5]$ & 0.027 \\
\hline
\end{tabular}

GSTM1 positive $(M 1+)$, GSTM1 null $(M 1-)$, GSTT1 positive $(T 1+)$ and GSTT1 null $(T 1-)$

$O R$ odds ratios, $\mathrm{Cl}$ confidence intervals. Wild genotypes were used as a reference (GSTM1 positive, GSTT1 positive)

Table 4 Correlation between GSTM1, GSTT1, CYP1A1 genotypes and different Gleason scores

\begin{tabular}{|c|c|c|c|c|}
\hline \multirow[t]{2}{*}{ Polymorphism } & \multicolumn{2}{|c|}{ Gleason score } & \multirow[t]{2}{*}{$\mathrm{OR} / \mathrm{Cl}$} & \multirow[t]{2}{*}{$P$ value } \\
\hline & $\begin{array}{l}\mathrm{GS} \leq 7 \\
(n=37)\end{array}$ & $\begin{array}{l}\mathrm{GS}>7 \\
(n=64)\end{array}$ & & \\
\hline \multicolumn{5}{|l|}{ GSTM1 } \\
\hline$M+$ & 17 (45.94\%) & $26(40.62 \%)$ & 0.89 [0.69-1.15] & 0.40 \\
\hline$M-$ & $20(54.05 \%)$ & $38(59.38 \%)$ & & \\
\hline \multicolumn{5}{|l|}{ GSTT1 } \\
\hline$T+$ & $26(70.27 \%)$ & $37(57.81 \%)$ & $0.91[0.70-1.17]$ & 0.47 \\
\hline$T-$ & $11(29.72 \%)$ & 27 (42.19\%) & & \\
\hline \multicolumn{5}{|l|}{ GSTM1/GSTT1 } \\
\hline Both present & $13(35.14 \%)$ & 17 (26.57\%) & & \\
\hline Either null & $17(45.94 \%)$ & $29(45.31 \%)$ & $0.92[0.71-1.18]$ & 0.52 \\
\hline Both null & 7 (18.92\%) & $18(28.12 \%)$ & $0.87[0.66-1.14]$ & 0.31 \\
\hline \multicolumn{5}{|l|}{ CYP1A1 } \\
\hline $\mathrm{TT}$ & $29(78.37 \%)$ & $53(82.21 \%)$ & $0.78[0.56-1.07]$ & 0.13 \\
\hline $\mathrm{TC}$ & $8(21.63 \%)$ & $11(17.19 \%)$ & & \\
\hline
\end{tabular}

Tables 4 and 5 present the relation between the studied polymorphisms and the Gleason score and clinical stages at diagnosis of $\mathrm{PCa}$.

The logistic regression analyses presented in Tables 4 and 5 showed no statistically significant correlations between the GSTM1, GSTT1, CYP1A1 genotypes and different Gleason scores and clinical stages at diagnosis of $\mathrm{PCa}$.

\section{Discussion}

This paper reports for the first time in Algeria the relation between the genetic polymorphism of three enzymes metabolizing xenobiotics (CYP1A1, GSTM1, GSTT1)
Table 5 Relation between GSTM1, GSTT1, CYP1A1 genotypes and clinical stages at diagnosis of PCa

\begin{tabular}{lllll}
\hline Polymorphism & \multicolumn{2}{l}{ Clinical stages } & OR/Cl & P value \\
\cline { 2 - 3 } & $\begin{array}{l}\text { Localized } \\
(\boldsymbol{n}=\mathbf{4 2})\end{array}$ & $\begin{array}{l}\text { Advanced } \\
(\boldsymbol{n}=\mathbf{5 9})\end{array}$ & \\
& & & & \\
\hline GSTM1 & $18(42.85 \%)$ & $25(42.38 \%)$ & $0.99[0.85-1.16]$ & 0.98 \\
M+ & $24(57.15 \%)$ & $34(57.62 \%)$ & & \\
M- & & & & \\
GSTT1 & $28(66.66 \%)$ & $35(59.32 \%)$ & $1.11[0.94-1.31]$ & 0.18 \\
T+ & $4(33.34 \%)$ & $24(40.68 \%)$ & & \\
T- & & & & 0.75 \\
GSTM1/GSTT1 & & & \\
Both present & $15(35.71 \%)$ & $15(25.42 \%)$ & & \\
Either null & $17(40.48 \%)$ & $29(49.15 \%)$ & $1.02[0.87-1.20]$ & 0.19 \\
Both null & $10(23.81 \%)$ & $15(25.43 \%)$ & $0.89[0.75-1.06]$ & \\
CYP1A1 & & & & \\
TT & $33(78.57 \%)$ & $49(83.05 \%)$ & $0.84[0.69-1.03]$ & 0.10 \\
TC & $9(21.43 \%)$ & $10(16.95 \%)$ & & \\
\hline
\end{tabular}

and susceptibility to developing prostatic cancer. The choice of these allelic variants is justified for each variant's potential as a marker of susceptibility for different types of cancer.

A total of 202 Algerian men were recruited and the association was analyzed between the case and control subjects. This present article shows the effect of the GSTM1, GSTT1 and CYP1A1 polymorphism on PCa risk in an Algerian population.

Prostate cancer is a multifactorial disease resulting from the combined effects of multiple environmental and genetic factors. Individual differences in the susceptibility to carcinogens play an essential role in the development of sporadic cancer. Several polymorphic genes encoding enzymes involved in the biotransformation of carcinogens have been studied as possible prostate cancer risk modifiers, including the cytochrome P450 (CYP) genes and GST system.

Numerous studies have been conducted to determine the association between the CYP1A1.GSTM1, GSTT1 polymorphisms and $\mathrm{PCa}$. However, the results are conflicting due to differences in the studied populations, various genetic backgrounds, ethnic and geographical variation and different exposures to diverse environmental risk factors. These variations have been linked to increased incidence or aggressiveness of prostate cancer.

In the present study, no links were detected between $\mathrm{PCa}$ and T3801C polymorphism. Our results are consistent with previous reports: Souiden et al. [20], Mandić et al. [21] found that there is no significant 
association between T3801C polymorphism of CYP1A1 and $\mathrm{PCa}$ in the Tunisian and Caucasian populations, respectively. In addition, the results published by $\mathrm{Li}$ et al. [22] revealed that no correlation between T3801C polymorphism of CYP1A land predisposition to $\mathrm{PCa}$.

Nevertheless, many studies diverge from our results: Bag et al. [10], Vijayalakshmi et al. [23], Shaik et al. [24] and $\mathrm{He}$ et al. [25] postulated a strong involvement of CYP1A1 polymorphism T3801C in PCa development.

The results of our study and other reports suggest that the biotransformation enzyme CYP1A1 T3801C is polymorphic in the prostate tissue. Also, CYP1A1 T3801C gene polymorphism is involved with elevated enzymatic activity and/or inducibility which can result in an accumulation of genotoxic compounds and consequently to cancerogenesis.

In the current study, GSTM1 wild and null genotypes were found, respectively, in 42.57 and $57.42 \%$ of PCa patients. A similarity was observed in the healthy controls (50.49\% wild type and $49.50 \%$ null type, respectively). These results indicated no statistically significant association between GSTM1-null genotype and the increased risk of PCa. The lack of significant association of homozygous GSTM1-null gene in our study is consistent with reports from studies in South India, Japanese, American and Africans populations [26]. Also, these results are in agreement with the results of Mallick et al. [27] and Souiden et al. [28] in a Caribbean of African descent and Tunisian populations, respectively.

Conversely, our finding is not consistent with Turkish, [29] Iranian [30], Chilean [31], Japanese [32] and North Indian [33] studies, where significant association was found between the GSTM1-null genotype and risk of sporadic prostate cancer.

A number of studies presented in a meta-analysis of Wei et al. [34] (combined results of 36 studies including 6202 cases and 8209 controls) and Zhong-Yang Wang et al. [35] (contained data from 6741 patients and 9053 controls) were marked an association between the GSTM1-null genotype and PCa risk in the overall population and Asians. These variations may be attributed to the underlying geographical and ethnic factors.

We also investigated the association of GSTT1 and PCa. The frequency of the GSTT1-null genotype was higher among patients (37.62\%) than the controls (22.77\%); the association was statistically significant $(\mathrm{OR}=2.03$, CI [1.06-3.99], $p=0.03)$.

In our study, there was an increased risk of PCa with the GSTT1-null genotype, as reported in the Tunisian and Korean populations [28, 36]. Thus, GSTT1-null genotypes were associated with more than threefold increased risk of PCa in Iranian men [30].
Forty-three reports represented in the meta-analysis of Yang et al. [19] were recruited data from 26,393 subjects (9934 cases and 16,459 controls). There was marked an association between the GSTT1-null genotype and prostate cancer risk in the overall population. However, published results of Zhou et al. [37] indicate that GSTT1null genotype is associated with PCa risk in Caucasians, but not in the overall population. On the contrary, other studies found no evidence of an association between GSTT1 polymorphism for an increased risk of PCa [38, 39].

In addition, GSTT1 deletion was not correlated with prostate cancer in Asian, Caucasian, Korean, African and American men [40]. Other more meta-analysis have given the same result $[41,42]$.

When the two genotypes were combined, we observed that the presence of the genotypes GSTM1-null/GSTT1null (double null) was associated with an increased risk for developing PCa. Similar results have been found in Iran [30].

In this context, we can suggest that the double mutated genotype may play an important role in the susceptibility of PCa. Dual null deletion of GSTM1 and GSTT1 was not associated with prostate cancer in studies of African descent, Brazilian and Caucasians [43]. Another metaanalysis showed no association of these two genes in the development of prostate cancer [44].

The genotypes studied in the present work were also correlated with histopathologic parameters: Gleason scores and clinical stages at diagnosis of prostate cancer. The stratified analysis of studied polymorphisms on grade/stage of the patients did not reveal any significant association suggesting that the genotypes are not associated with the stage or aggressiveness.

Lima et al. [45], Rodrigues et al. [46] did not observe any association with GST and CYP1A1 genotypes and parameters of aggressiveness at diagnosis.

On the other hand, Safarinejad et al. [30] demonstrated that GSTM1-null and GSTT1-null genotype was observed in a higher frequency in patients with a Gleason score $>7$.

Since the GSTM1-null genotype is more frequent than GSTT1-null, this indicates that loss of function of GSTT1 has a more deleterious effect than GSTM1. In addition, GSTM1 and GSTT1 polymorphisms could cause disparities in enzyme activities, and GSTM1-null/GSTT1-null individuals have a complete absence of activity of these enzymes. In addition, individuals with homozygous deletions of GSTM1 or GSTT1 lack glutathione S-transferase and therefore may be unable to eliminate electrophilic carcinogens as efficiently, which may increase the risk of somatic mutations leading to tumor formation [47]. 
There was no significant association between the GSTM1, GSTT1, CYP1A1 genotypes and the clinicopathologic factors of prostate cancer. To better understand the role of these variants and to study their predictive value, tumor prognostic criteria should be examined, such as cancer-specific survival and overall survival.

The introduction of new molecular biomarkers such as GSTs and CYPs in the management of patients with PCa may improve their clinical results.

Our study has some limitations. First, the sample size for genetic analysis is relatively small. Therefore, further studies should be done on a larger scale for confirmation of our results and functional studies undertaken to explore the effect of GST and CYP variants.

\section{Conclusions}

This study presents the first data on the frequency of CYP1A1 (T3801C), GSTM1 and GSTT1 polymorphisms in an Algerian population. We did not find significant associations between genetic polymorphisms (CYP1A1 T3801C genotypes and GSTM1-null) and PCa. However, we have indicated that GSTT1-null and GSTM1/ GSTT1-double null are associated with increased risk of prostate cancer.

\section{Abbreviations}

CYP: cytochrome P450; DRE: digital rectal examination; GSTA: human glutathione S-transferase alpha; GSTK: glutathione S-transferase kappa; GSTM: glutathione S-transferase mu; GSTO: glutathione S-transferase omega; GSTP: glutathione S-transferase pi; GSTS: glutathione S-transferase sigma; GSTT: glutathione S-transferase theta; GSTZ: glutathione S-transferase zeta; PCR-RFLP: polymerase chain reaction restriction fragment length polymorphism; PSA: prostate-specific antigen; XME: xenobiotic-metabolizing enzymes.
\end{abstract}

\section{Acknowledgements}

The authors acknowledge Pr. Abderrezak Dahdouh and all the staff of the Department of Urology and Renal Transplantation," for their help and support.

\section{Authors' contributions}

SM gave idea and contributed to protocol/project development, data collection or management, data analysis and manuscript writing/editing. DR contributed to protocol/project development, manuscript writing/editing and final correction of the manuscript. TK and MC contributed to data analysis. NA contributed to protocol/project development. DS contributed to protocol/ project development and final correction of the manuscript. They all approved the final version of the manuscript.

\section{Funding}

This study had no funding from any resource.

\section{Availability of data and material}

The datasets used and/or analyzed during the current study are available from the corresponding author on reasonable request.

\section{Ethics approval and consent to participate}

The ethics committee of the Dr. BENBADIS - Constantine University Hospital Centre has approved the study; Ethical approval number 03. The use of human blood sample and the protocol in this study strictly conformed to the principles expressed in the Declaration of Helsinki. We work in accordance with the Declaration of Helsinki (1964): Ethical principles applicable to medical research on human subjects and in accordance with the recommendations of the Algerian national council for ethics in health sciences. Informed consent (written) was obtained from all participants.

\section{Consent for publication}

All patients included in this research gave written informed consent to publish the data contained within this study.

\section{Competing interests}

The authors declare that they have no competing interests.

\section{Author details}

${ }^{1}$ Department of Animal Biology, Laboratory of Molecular and Cellular Biology, Faculty of Natural and Life Sciences, University of Frères Mentouri Constantine 1, 25000 Constantine, Algeria. ${ }^{2}$ Department of Medicine, Laboratory of Biology and Molecular Genetics, University Salah Boubnider Constantine 3, 25000 Constantine, Algeria. ${ }^{3}$ Innovation Academy, 43000 Mila, Algeria.

Received: 10 October 2019 Accepted: 14 July 2020

Published online: 18 September 2020

References

1. Foley R, Hollywood D, Lawler M (2004) Molecular pathology of prostate cancer: the key to identifying new biomarkers of disease. Endocr Relat Cancer 11(3):477-488

2. Crawford ED, Higano CS, Shore ND, Hussain M, Petrylak DP (2015) Treating patients with metastatic castration resistant prostate cancer: a comprehensive rev available therapies. J Urol 194(6):1537-1547

3. Hamdi CM, Bidoli E, Birri S et al (2015) Cancer estimation of incidence and survival in Algeria 2014. J Cancer Res Ther 3(9):100-104

4. Hoffman RM (2011) Clinical practice: screening for prostate cancer. N Engl J Med 365(21):2013-2019

5. Sutcliffe S, Colditz GA (2013) Prostate cancer: is it time to expand the research focus to early-life exposures. Nat Rev Cancer 13(3):208-518 (discussion Nat Rev Cancer. 2013 May;13(5):376)

6. Gong M, Dong W, Shi Z, Xu Y, Ni W, An R (2012) Genetic Polymorphisms of GSTM1, GSTT1, and GSTP1 with prostate cancer risk: a meta-analysis of 57 studies. PLOS ONE 7(11):1.e50587-12.e50587

7. Cerliani MB, Pavicic W, Gili JA, Klein G, Saba S, Richard S (2016) Cigarette smoking, dietary habits and genetic polymorphisms in GSTT1, GSTM1 and CYP1A1 metabolic genes: a case-control study in oncohematological diseases. World J Clin Oncol 7(5):395-405

8. Corchero J, Pimprale S, Kimura S, Gonzalez FJ (2001) Organization of the CYP 1A cluster on human chromosome 15: implications for gene regulation. Pharmacogenetics 11(1):1-6

9. Masson LF, Sharp L, Cotton SC, Little J (2005) Cytochrome P-4501A1 gene polymorphisms and risk of breast cancer: a HuGE review. Am J Epidemiol 161(10):901-915

10. Bag A, Jyala NS, Bag N (2015) Cytochrome P450 1 A1 genetic polymorphisms as cancer biomarkers. Indian J Cancer 52(4):479-489

11. Board PG, Menon D (2013) Glutathione transferases, regulators of cellular metabolism and physiology. Biochim Biophys Acta 1830(5):3267-3288

12. Galal AM, Walker LA, Khan IA (2015) Induction of GST and related events by dietary phytochemicals: sources, chemistry, and possible contribution to chemoprevention. Curr Top Med Chem 14(24):2802-2821

13. Sharma A, Gupta S, Sodhani P, Singh V, Sehgal A, Sardana S et al (2015) Glutathione S-transferase M1 and T1 polymorphisms, cigarette smoking and HPV infection in precancerous and cancerous lesions of the uterine cervix. Asian Pac J Cancer Perv 16(15):6429-6438

14. Zmorzyński S, Świderska-Kołacz G, Koczkodaj D, Filip AA (2015) Significance of polymorphisms and expression of enzyme-encoding genes related to glutathione in hematopoietic cancers and solid tumors. Biomed Res Int. 2015:853573

15. Oakley A (2011) Glutathione transferases: a structural perspective. Drug Metab Rev 43(2):138-151

16. Peddireddy V, Badabagni SP, Gundimeda SD, Mamidipudi V, Penagaluru PR, Mundluru HP (2016) Association of CYP1A1, GSTM1 and GSTT1 gene 
polymorphisms with risk of non-small cell lung cancer in Andhra Pradesh region of South India. Eur J Med Res 18(21):17

17. Economopoulos KP, Sergentanis TN, Vlahos NF (2010) Glutathione S-transferase $\mathrm{M} 1, \mathrm{T1}$, and $\mathrm{P} 1$ polymorphisms and ovarian cancer risk: a meta-analysis. Inter J Gynecol Cancer 20:732-737

18. Huang W, Shi H, Hou Q, Mo Z, Xie X (2015) GSTM1 and GSTT1 polymorphisms contribute to renal cell carcinoma risk: evidence from an updated meta-analysis. Sci Rep 14(5):17971

19. Yang Q, Du J, Yao X (2013) Significant association of glutathione S-Transferase T1 null genotype with prostate cancer risk: a meta-analysis of 26,393 subjects. PLoS ONE 8(1):e53700

20. Souiden Y, Mahdouani M, Chaieb K, Bakhrouf A, Mahdouani K (2012) Lack of association of CYP1A1 polymorphism with prostate cancer susceptibility of Tunisian Men. Genet Test Mol Biomark 16(7):661-666

21. Mandić S, Horvat V, Marczi S, Lukić I, Galić J (2014) Association study of cytochrome P450 1A1*2A polymorphism with prostate cancer risk and aggressiveness in croatians. Coll Antropol 38(1):141-146

22. Li H, Xiao D, Hu L, He T (2012) Association of CYP1A1 polymorphisms with prostate cancer risk: an updated meta-analysis. Mol Biol Rep 39(12):10273-10284

23. Vijayalakshmi K, Vettriselvi V, Krishnan M, Shroff S, Jayanth VR, Paul SF (2005) Cytochrome P 4501A1 gene variants as susceptibility marker for prostate cancer. Cancer Biomark 1(4-5):251-258

24. Shaik AP, Jamil K, Das P (2009) CYP1A1 polymorphisms and risk of prostate cancer. Urol J 6(2):78-86

25. He XF, Wei W, Liu ZZ, Shen XL, Yang XB, Wang SL et al (2014) Association between the CYP1A1 T3801C polymorphism and risk of cancer: evidence from 268 case-control studies. Gene 534(2):324-344

26. Caceres DD, Iturrieta J, Acevedo C, Huidobro C, Varela N, Quinones L et al (2005) Relationship among metabolizing genes, smoking and alcohol used as modifier factors on prostate cancer risk: exploring some genegene and gene-environment interactions. Eur J Epidemiol 20(1):79-88

27. Mallick S, Romana M, Blanchet P, Multigner L (2007) GSTM1 and GSTT1 polymorphisms and the risk of prostate cancer in a Caribbean population of African descent. J Urol 69(6):1165-1169

28. Souiden Y, Mahdouani M, Chaieb K, Elkamel R, Mahdouani K (2010) Polymorphisms of glutathione-S-transferase $\mathrm{M} 1$ and $\mathrm{T} 1$ and prostate cancer risk in a Tunisian population, Tunisia. Cancer Epidemiol 34(5):598-603

29. Silig Y, Pinarbasi H, Gǔnes S, Ayan S, Bagci H, Cetinkaya O (2006) Polymorphisms of CYP1A1, GSTM1, GSTT1, and prostate cancer risk in Turkish population. Cancer Invest 24(1):41-45

30. Safarinejad MR, Shafiei N, Safarinejad SH (2011) Glutathione S-transferase gene polymorphisms (GSTM1, GSTT1, GSTP1) and prostate cancer: a case-control study in Tehran, Iran. Prostate Cancer Prostatic Dis 14(2):105-113

31. Acevedo C, Opazo JL, Huidobro C, Cabezas J, Iturrieta J, Quinones Sepulveda L (2003) Positive correlation between single or combined genotypes of CYP1A1 and GSTM1 in relation to prostate cancer in Chilean people. Prostate 57(1):111-117

32. Murata $M$, Watanabe $M$, Yamanaka $M$, Kubota $Y$, Ito $H$, Nagao $M$ et al (2001) Genetic polymorphisms in cytochrome P450 (CYP) 1A1, CY1A2 CYP2E1, glutathione transferases (GST) M1, GSST1, and susceptibility to prostate cancer in the Japanese population. Cancer Lett 165(2):171-177
33. Srivastava D, Mandhani A, Mittal B, Mittal RD (2005) Genetic polymorphism of glutathione S-transferase genes (GSTM1, GSTT1 and GSTP1) and susceptibility to Prostate cancer in Northern India. BJU Int 95(1):170-173

34. Wei B, Xu Z, Zhou Y, Ruan J, Cheng H, Xi B et al (2012) Association of GSTM1 Null allele with prostate cancer risk: evidence from 36 case-control studies. PLOS ONE 7(10):e46982

35. Wang ZY, Li HY, Jiang Z, Zhou TB, Drummen GP (2016) GSTM1 Gene polymorphism is implicated in increased susceptibility to, prostate cancer in Caucasians and Asians. Technol Cancer Res Treatment 15(6):69-78

36. Shin YE, Hwang IW, Jin HJ (2016) Association between glutathione S-transferases $\mathrm{M} 1, \mathrm{~T} 1$ and $\mathrm{P} 1$ gene polymorphisms and prostate cancer in Koreans. Genes Genom 38(2):235-241

37. Zhou TB, Drummen GPC, Jiang DZ, Qin YH (2014) GSTT1 polymorphism and the risk of developing prostate cancer. Am J Epidemiol 180(1):1-10

38. Kumar V, Yadav CS, Datta SK, Singh S, Ahmed RS, Goel S et al (2011) Association of GSTM1 and GSTT1 polymorphism with lipid peroxidation in benign prostate hyperplasia and prostate cancer: a pilot study. Dis Mark 30(4):163-169

39. Kwon DD, Lee JW, Han DY, Seo IY, Park SC, Jeong JH et al (2011) Relationship between the glutathione-S-transferase P1, M1, and T1 genotypes and prostate cancer risk in Korean subjects. Korean J Urol 52(4):247-252

40. Cai Q, Wang Z, Zhang W, Guo X, Shang Z, Jiang N et al (2014) Association between glutathione $\mathrm{S}$ - transferases $\mathrm{M} 1$ and $\mathrm{T} 1$ gene polymorphisms and prostate cancer risk: a systematic review and meta-analysis. Tumour Biol 35(1):247-256

41. Mo Z, Gao Y, Cao Y, Gao F, Jian L (2009) An updating meta-analysis of the GSTM1, GSTT1, and GSTP1 polymorphisms and prostate cancer: a HuGe review. Prostate 69(6):662-688

42. Wang J, Xu Y, Fu Q, Yu J, Chen Z, Liu Z et al (2013) Association of GSTTI gene polymorphisms with the risk of prostate cancer: an updating metaanalysis. Tumor Biol 34(3):1431-1440

43. Lavender NA, Benford ML, VanCleave TT, Brock GN, Kittles RA, Moore JH et al (2009) Examination of polymorphic glutathione S-transferase (GST) genes, tobacco smoking and prostate cancer risk among men of African descent: a case-control study. BMC Cancer 16(9):397

44. Ntais C, Polycarpo A, loannidis JP (2005) Association of GSTM1, GSTT1, and GSTP1 gene polymorphisms with the risk of prostate cancer: a metaanalysis. Cancer Epidemiol Biomark Prev 14(1):176-181

45. Lima MM Jr, Oliveira MN, Granja F, Trindade AC, De Castro Santos LE, Ward LS (2008) Lack of association of GSTT1, GSTM1, GSTO1, GSTP1 and CYP1A1 polymorphisms for susceptibility and outcome in Brazilian prostate cancer patients. Folia Biol (Praha) 54(3):102-108

46. Rodrigues IS, Kuasne H, Losi-Guembarovski R, Fuganti PE, Gregório EP, Kishima MO et al (2011) Evaluation of the influence of polymorphic variants CYP1A1*2B, CYP1B1*2, CYP3A4*1B, GSTM1*0, and GSTT1*0 in prostate cancer. Urol Oncol 29(6):654-663

47. Coughlin SS, Hall IJ (2002) A review of genetic polymorphisms and prostate cancer risk. Ann Epidemiol 12(3):182-196

\section{Publisher's Note}

Springer Nature remains neutral with regard to jurisdictional claims in published maps and institutional affiliations. 